\title{
IRMHILD BARZ
}

\section{Interferenzen beim Wortschatzausbau}

Zum Zusammenspiel verschiedener Nominationsverfahren

\begin{abstract}
Der Beitrag untersucht auf der Grundlage eines zeichentheoretischen Ansatzes verschiedene Verfahren der Benennungsbildung in ihrem Zusammenwirken bei lexikalischen Innovationen. Erörtert werden die Interferenz zwischen natürlicher und künstlicher Benennungsbildung sowie die Interferenz zwischen Wortbildung und Bedeutungsbildung. Welche Interferenztypen auftreten und mit welchen Folgen für Struktur und Bedeutung der lexikalischen Innovation sie in der gegenwärtigen Wortschatzentwicklung wirksam sind, wird an Fallbeispielen illustriert.
\end{abstract}

\section{Anliegen}

Die nominationstheoretisch orientierte Lexikforschung stellt sich unter anderem die Aufgabe, verschiedene Verfahren der Benennungsbildung (= Nominationsverfahren), und zwar Wortbildung, Bedeutungsbildung und Phraseologisierung, nach ihrem quantitativen und qualitativen Anteil am Wortschatzausbau zu untersuchen (Fleischer 1989, S. 16). Bei den ,natürlichen" Nominationsverfahren, wie man Wortbildung, Bedeutungsbildung und Phraseologisierung zusammenfassend nennen könnte, handelt es sich jeweils um diskrete Bildungsmöglichkeiten, die zu formal und/oder semantisch spezifischen Benennungen führen, nämlich zu sekundären Wörtern, zu neuen Bedeutungen oder zu Phraseologismen. Neue Benennungen entstehen jedoch nicht nur durch Wortbildung oder Bedeutungsbildung oder Phraseologisierung, sondern auch durch Wortbildung zusammen mit Bedeutungsbildung (Gesundheitstelefon) oder durch Phraseologisierung zusammen mit Bedeutungsbildung (den Hut aufhaben). Bei der Phraseologisierung ist das Gebundensein an die Bedeutungsbildung geradezu konstitutiv für einen zentralen Bereich, für die figurative Phraseologisierung (Munske 1993; Fleischer 1997; Burger 1998). Von daher bliebe die Modellierung lexikalischer Innovationen unvollständig, würden nicht - gewissermaßen grenzüberschreitend - auch Überlappungen zwischen diesen Nominationsverfahren in den Blick genommen werden.

Grenzen sind in der Nominationsforschung schließlich noch in einer anderen Richtung zu überschreiten, und zwar wenn Firmen-, Produkt-, Mar- 
ken- und Dienstleistungsnamen („Ökonyme“, Platen 1997, S. 14) als Analyseobjekte einbezogen werden. Deren Bildung kann mit den Modellen der Wortbildung, Bedeutungsbildung und Phraseologisierung nicht hinreichend beschrieben werden. Hier kommt das Zusammenwirken mit der „Urschöpfung“ (Henzen 1965, S. 5), einem „künstlichen“ Nominationsverfahren, ins Spiel. Zahlreiche Ökonyme können als eine Verbindung von KunstwortSegmenten mit natürlichsprachlichen Einheiten angesehen werden, vgl. etwa die folgenden Namen für Computerschulen: Compu-Net (München), Comtrix (Langenhagen), Comtech (Köln) oder Firmennamen aus diesem Bereich: Ascom, Telekom (Computer Bild 22/1997).

Sucht man in Lexikologie und Wortbildung nach Beschreibungskategorien für das Zusammenspiel zwischen natürlichen und künstlichen Nominationsverfahren und zwischen den natürlichen Nominationsverfahren, wird man bislang kaum fündig.

Zwischen der objektsprachlichen Verfahrensvielfalt bei der Bildung von Benennungen einerseits und dem verfügbaren Kategorieninventar für deren Beschreibung andererseits besteht insofern eine Diskrepanz, als wir mit der Differenzierung zwischen Wortbildung, Phraseologisierung, Bedeutungsbildung und Urschöpfung Beschreibungsgrenzen ziehen, die das Interferieren der Verfahren als Beschreibungsgegenstand weder nahe legen noch ausdrücklich vorsehen. Was der Textlinguistik mit ihrem Konzept der Mustermischung gelingt, nämlich Mischtypen systematisch zu erfassen (Lerchner 1990, S. 262), hat die Nominationsforschung in Bezug auf die Beschreibung und Kategorisierung der Benennungsbildung weitgehend noch $\mathrm{zu}$ leisten.

Meine Ausführungen sollen ein Beitrag zur Lösung dieser Aufgabe sein. Statt des Nebeneinanders der Nominationsverfahren sollen Fälle ihres Miteinanders bzw. Ineinanders, das ich Interferenz nennen möchte (vgl. 2.), in den Mittelpunkt gerückt werden. Zwei Arten von Interferenzen werden behandelt, zum einen die Interferenz zwischen natürlicher Benennungsbildung und Urschöpfung, zum anderen die zwischen den natürlichen Verfahren Wortbildung und Bedeutungsbildung. Dass das nur ein kleiner Ausschnitt aus der Vielfalt von Interferenzerscheinungen sein kann, liegt auf der Hand. Wir gewinnen zum Beispiel außerdem neue Wörter oder neue Bedeutungen von Wörtern oder beleben alte Wörter wieder durch die Reduktion von Phraseologismen (Fettnäpfchen), wir implizieren in die Wortstruktur zunehmend Einheiten mit Phrasenstruktur (das Jahr-2000-Problem, Jahr2000-fähig) oder bilden aus Phraseologismen Wörter (sich die größten Rosinen aus dem Kuchen herauspicken > Rosinenpickerei, Vier-AugenGespräch, das Inkrafttreten). Diese Phänomene sind in ihrem Anteil an Wortschatzinnovationen der Gegenwartssprache bislang ebenfalls nicht hinreichend beschrieben. 


\section{Begriffliches}

Die Nominationsforschung gilt als noch nicht etablierte oder zumindest in Bezug auf ihren Gegenstand als schwach konturierte Forschungsrichtung. Entsprechend unverbindlich bzw. heterogen bietet sich ihre Terminologie dar (Knobloch/Schaeder 1996, S. 7). Für mein Anliegen wähle ich die folgenden Begriffsbestimmungen:

2.1 Den Terminus Nomination gebrauche ich zeichentheoretisch im Sinne von Lerchner als ,zeichenhaft konstituierte[n] Bezug von Signifikaten [...] auf (Komplexe von) sprachspezifischen Signifikanten" (Lerchner 1997, S. 153) ${ }^{1}$, im Sinne eines Signifikationsprozesses also, bei dem es um die Versprachlichung von Konzepten geht. Die verschiedenen Ausprägungen der Bezugnahme sind die Nominationsverfahren. Deren Produkte stellen die Nominationseinheiten oder (synonymisch) Benennungen dar. Nach den jeweils am Signifikationsprozess beteiligten Zeichenkomponenten ergibt sich eine Ordnung in semantische (Wandel des Signifikats), morphosemantische (Wandel von Signifikant und Signifikat) und morphologische Prozesse (nur Signifikant ist betroffen), wie sie Munske (1990; vgl. auch Lipka 1994, S. 6) ausführlich darstellt. Wenn man die ökonymischen Benennungen mit einbeziehen will, greift diese Definition allerdings zu kurz. Das Problem ergibt sich auf Grund der Qualifizierung der Signifikanten als ,sprachspezifisch“. Da Ökonyme auch übereinzelsprachliche Geltung haben können, müssen sie hier zunächst als Sonderfall ausgewiesen werden.

Die terminologische Differenzierung zwischen natürlicher und künstlicher Benennungsbildung folgt der tradierten Unterscheidung von Wortbildung und Wortschöpfung. Und zwar insofern in einem erweiterten Sinn, als auch Mehrworteinheiten als Produkte der Urschöpfung ausdrücklich einbezogen werden. Unter natürlicher Benennungsbildung werden, wie bereits gesagt, Wortbildung, Phraseologisierung und Bedeutungsbildung zusammengefasst. ${ }^{2} \mathrm{Zu}$ natürlicher Benennungsbildung sind Sprecher auf Grund ihrer Kompetenz in der Lage (Wellmann 1998, S. 409; Eichinger 1997, S. 25). Sie bilden aus bedeutungstragenden sprachlichen Einheiten unbewusst oder bewusst neue Benennungen oder neue Bedeutungen. Zum Zeitpunkt der Prägung sind diese Innovationen strukturell durchschaubar und morphosemantisch oder semantisch motiviert, weil sie auf vorhandenem Material beruhen.

Bei der Begriffsbestimmung für die künstliche Benennungsbildung berufe ich mich auf Henzen: „Wort- oder Urschöpfung nennen wir die Entstehung eines Wortes aus einer Lautgruppe, ohne daß die mit ihr zu verbindende Vorstellung durch eine verwandte, schon an sie geknüpfte Vorstellung

${ }^{1}$ Vgl. in diesem Sinn auch Motsch 1999, S. 20; Wellmann 1998, S. 409; Eichinger 1997, S. 25 .

2 Kategorisierung der Phraseologisierung und Bedeutungsbildung hier nach Munske 1990 und 1993. 
vermittelt ist (z. B. töff für Motorrad)“ (Henzen 1965, S. 5; vgl. auch Erben 1993, S. 18; Naumann 1986, S. 3 ff.). Künstliche Benennungen bzw. Benennungskonstrukte sind demnach Zeichen, bei denen morphosemantische oder semantische Motivation kaum gegeben sein kann. Der „Konstrukteur“ folgt nicht erworbenen Automatismen und verwendet auch nicht bekannte sprachliche Zeichen, sondern er kreiert die Benennungen willkürlich und experimentiert mit verschiedenartigen Elementen und Strukturen ${ }^{3}$ (Twingo, Eduscho, elmex, Tam-Com 215 [Anrufbeantworter von Bosch]). Für diese Nomination sind auch die anschaulichen Termini Worterfindung (Matussek 1994), Wortfabrikation und Namenkonstruktion vorgeschlagen worden (Seebold (1981, S. 192; Stoll 1999; vgl. auch Koß 1992, S. 145 f.).

Benennungskonstrukte kommen hauptsächlich als Ökonyme vor (dazu 3.), einer Benennungsklasse zwischen Appellativa und Eigennamen (Janich 1999, S. 50). Deren diasystematische und funktionale Besonderheiten bedingen eine ganz spezifische formale und semantische Prägung. Sie steht vor allem im Dienst von Unverwechselbarkeit, Merkbarkeit und Werbewirksamkeit. Der Umkehrschluss, dass alle Ökonyme auch Benennungskonstrukte seien, gilt nicht. Für eine detaillierte Klassifizierung sei auf Platen 1997 verwiesen. ${ }^{4}$

2.2 Wie in 1. bereits angedeutet, verwende ich den Terminus Interferenz abweichend von der in der Sprachkontaktforschung üblichen Bedeutung. Er steht hier für das Ineinandergreifen von Nominationsverfahren, und zwar bei der Erzeugung ein und derselben Benennung. Unterschieden wird zwischen gradueller und totaler Interferenz.

Bei der graduellen Interferenz mischen sich verschiedene Merkmale zweier Verfahren so, dass Produkte der Randbereiche der jeweiligen Kategorien entstehen. Anders gesagt, die Produkte stellen Schnittstellen zweier Kategorien dar. So tritt bei Jogurette (Markenname für ein Schokoladenprodukt mit Joghurt) ein aus der natürlichen Sprache stammendes Suffix an eine „künstliche“ (verfremdend gekürzte) Basis. Das Wort entspricht wegen dieser Basis nur bedingt dem fraglichen Derivationsmodell (Operette); es hat sowohl künstliche als auch natürliche Züge. Die graduelle Interferenz ist charakteristisch für die Bildung von Ökonymen.

Bei der totalen Interferenz sind zwei Verfahren simultan ,in Kombination“ an der Bildung einer Benennung beteiligt, wie z. B. Bedeutungsbildung und Komposition in Salamitaktik, Taktik, [politische] Ziele durch kleinere Forderungen und entsprechende Zugeständnisse von der Gegenseite zu erreichen suchen' (Duden 1999, S. 3272). Die metaphorische Bedeutung von

${ }^{3}$ Bei den Ökonymen allerdings im Rahmen juristischer und pragmatischer Grenzen (Stoll 1999, S. 55).

4 Platen (1997) unterscheidet in der formalen Bildung der Ökonyme drei Klassen, die Übernahmen, d. h. die Onymisierung vorhandener Appellativa (Golf für einen Autotyp), außerdem deformierte (Schauma), derivierte (Lemocin), zusammengesetzte (Dentagard) und komplexe Konzeptformen (Du darfst) sowie Kunstwörter (elmex). 
Salami ist an die Kombination des Wortes mit einem substantivischen Zweitglied gebunden.

Von der Interferenz zu unterscheiden sind zwei weitere, nominationstheoretisch ebenfalls bedeutsame Relationen, zum einen die Konkurrenz (Fleischer 1992, S. 53 ff.) zwischen den Verfahren, die bei bedeutungsähnlichen, aber verschieden strukturierten homolexikalen Dubletten vorliegt (schwarzer Markt - Schwarzmarkt, schwarze Kassen - Schwarzkassen, rot werden erröten), zum anderen die Konvergenz, die das Zusammenwirken verschiedener Benennungsverfahren bei der Bildung heterolexikaler Benennungen mit einer semantischen Invariante bezeichnet (,Vergleich': mehlig, seidenweich, hart wie Stein).

2.3 Für die bzw. eine lexikalische Bedeutung einer Benennung verwende ich zur Abgrenzung der Bedeutung von anderen den Terminus Semem, und zwar nur im Sinn der Abgrenzung, nicht im strukturalistischen Sinn einer vollständigen Modellierung von Bedeutung (Blank 1997, S. 58). Das Signifikat entspricht einem Teil des einzelsprachlichen Bedeutungswissens der Sprecher. Mit dem Zeichen als Ganzheit sind außerdem weitere Wissensebenen assoziativ verknüpft. Diese Bestimmung soll für usuelle und für okkasionelle Benennungen gleichermaßen gelten. Warum in der Nominationsforschung prinzipiell auch Okkasionalismen Berücksichtigung finden sollten, deren Beitrag zum Wortschatzausbau sich jeweils erst herausstellen muss, begründet GroBe am Beispiel historischer lexikalischer Einheiten. Sie können für die Nominationstheorie ,von Belang sein, weil solche Bildungen und Bezeichnungen die im Wortschatz einer Kommunikationsgemeinschaft angelegten Potenzen aufzeigen und Wege der Konzeptualisierung andeuten" (Große 1997, S. 19).

\section{Natürliche und künstliche Benennungsbildung}

3.1 Durch Interferenz zwischen Benennungsbildung und Urschöpfung ist ein großer Teilbereich der Ökonymenbildung gekennzeichnet. Ökonyme und verwandte Konstrukte seien daher zunächst ganz knapp als spezifische Wortschatzeinheiten charakterisiert.

Die sprachlichen Artefakte, wie Kalverkämper (1978, S. 302) die Ökonyme nennt, sind in der Gegenwart keineswegs Marginalien, sondern sie strömen massenhaft auf die Sprachbenutzer ein, auch wenn sie meist nicht aktiv gebraucht werden. Sie begegnen insbesondere in den Wirtschaftsbereichen Produkt- und Dienstleistungswerbung, Informatik, Telekommunikation, Bahn- und Autoverkehr sowie Reise und Touristik. 1999 gibt das Deutsche Patentamt in München den aktuellen Bestand an eingetragenen Markennamen mit ca. einer halben Million an, die jährliche Zuwachsrate mit 70000 (Platen 2000, S. 241).

Neben den Firmen- und Produktnamen gehören zu onymischen Artefakten auch konstruierte, „erfundene“ Benennungen in der Elektronik, ohne die 
man im Bereich der realen und virtuellen Kommunikationsmöglichkeiten über und mit Computer und Internet nicht mehr auskommt. Technische Neuerungen, neue Adressen, Passwörter und Programmnamen bieten nicht nur reichlich Gelegenheit, neue sprachliche Artefakte zu rezipieren, sondern sie zwingen die Benutzer auch, bei der Speicherung von Dateien, der Herstellung von Verzeichnissen o. Ä. code- und namenerfindend aktiv zu werden. Oft bestimmen technisch bedingte Vorschriften die zu wählenden Formen. Für die Benennung von sog. Makros, kleinen Arbeitsprogrammen, die der Computernutzer selbst erstellt, wird beispielsweise Folgendes vorgeschrieben: „Der Name darf bis zu 80 Zeichen lang sein und muß mit einem Buchstaben beginnen, Ziffern sind erlaubt, sofern sie nicht am Anfang des Namens stehen; Leerzeichen dürfen nicht verwendet werden. Nutzen Sie statt dessen die Groß- und Kleinschreibung, um mehrere Begriffe zusammenzusetzen" (Computer Bild 22/1997, S. 120). Die Ergebnisse und Folgen dieser Taufakte liegen auf der Hand. Es wird - ganz im Sinne der Vorschriften willkürlich experimentiert mit Buchstabenverbindungen und Wortfolgen. Hauptziele sind die Unikalität des Namens und seine Computer-Tauglichkeit. Für Grenzziehungen zwischen monolexikaler und polylexikaler Einheit wie bei den natürlichen Benennungen besteht keine technische Möglichkeit und auch keine Notwendigkeit; die Grenzen verschwimmen.

Wortbildungshand- und -lehrbücher nehmen sich der Benennungsartefakte bisher kaum an. Die Abschnitte zur onymischen Wortbildung in Fleischer/ Barz (1995) beschränken sich $z$. B. weitgehend auf Personen- und geographische Namen.

Kommt schon Personen- und geographischen Namen eine Sonderstellung im Wortschatz zu, aus der Spezifika für die Wortbildung erwachsen, so gilt das für Ökonyme und artverwandte Namen in der Informatik - den beiden Namengruppen mit genetischen und funktionalen Eigenheiten - noch verstärkt.

3.2 Was die monolexikalen Ökonyme angeht ${ }^{5}$, so lassen sich in vorliegenden Strukturuntersuchungen zwei Vorgehensweisen erkennen. Während sich Stoll (1999) in seiner Typologie bemüht, die Ökonyme ihrer formalen Struktur nach weitgehend in das natürlichsprachliche Wortbildungssystem einzubauen, arbeiten Platen (1997) und Janich (1999) sehr deutlich eine Spezifik der Bildungsweisen heraus. Letzteres wird der Konstruktion von Ökonymen, wie ich meine, besser gerecht.

Am Beispiel von komplexen Medikamentennamen mit sog. kommerziellen Suffixen (Koß 1990, S. 102 unter Berufung auf Voigt 1984) wie z.B. -lon, -i, -al, -ol, -il, -an, -on, -in, -en, -at, -it, -ax, -ox soll daher im Folgenden die Bildung von Ökonymen im Vergleich mit der Wortbildung als ein Verfahren eigenen Typs, als Schnittstelle zwischen den natürlichen und den künstlichen Nominationsverfahren bestimmt werden.

${ }^{5}$ Zu den Termini polylexikal und monolexikal vgl. Gréciano 1997, S. 169. 
Für den Vergleich mit der Wortbildung sind zunächst die morphosemantischen Eigenschaften der Segmente der komplexen Struktur relevant. Mit den natürlichen Suffixen haben die kommerziellen Suffixe gemeinsam, dass sie in Kombination mit anderen Segmenten rekurrent und positionsfest auftreten. Wie Fremdsuffixe sind die meisten betont. Sie kategorisieren die Wörter als Substantive. Semantisch bleiben sie weitgehend unbestimmt, nur aus Erfahrung mit anderen Namen (oder selbstverständlich bei paralleler Präsenz von Name und bezeichneter Sache) kann der Rezipient das benannte Denotat einem Sachbereich zuweisen bzw. das Suffix semantisch ,,aufladen“ (Platen 1997, S. 79). In Lemocin, L-Thyroxin, Sanopin, Aspirin, Veronal, Faustan verweisen die Suffixe auf den Bereich ,Medikament', jedoch nicht zwingend, wie die Namen für andere Stoffe unterstreichen: Biskin für ein Fett, domal für ein Reinigungsmittel, softlan für ein Weichspülmittel. Anders als bei der Polyfunktionalität natürlicher Suffixe (vgl. -er: ,Person', ,Gerät' u. a.) ist der Bezug kommerzieller Suffixe nicht auf bestimmte Denotatsklassen beschränkt. Sie können ganz willkürlich beliebige Denotate benennen. Wenn ich richtig sehe, unterliegen sie auch in Bezug auf die phonologisch-morphologische Gestalt der Basis keinerlei Distributionsbeschränkungen. Was Künstlichkeit und referentielle Unbestimmtheit dieser Bildungen mit verursacht, ist die Art der Basis. Die Basen sanop, lemo, aspir, L-Thyrox, Bisk in den o.g. Beispielen sind singuläre, nicht rekurrente, nicht wortfähige Elemente. ${ }^{6}$ Ihre Bedeutung ist, wenn überhaupt vorhanden, meist nur mit Fremdsprachenkenntnissen und allenfalls andeutungsweise erkennbar. Für kommerzielle Suffixe lassen sich demnach keine morphologisch oder semantisch bestimmten $\mathrm{Ba}$ sisklassen angeben (Eisenberg 1998, S. 213); die Bildungen sind als Ganzes nicht modellierbar. Der Ökonymenstatus lässt offenbar aus funktionalen Gründen die Erzeugung nach Mustern kaum zu. Insofern verwundert es nicht, dass der Bereich mit deutlicher Neigung zu Suffixrekurrenz bei Ökonymen die in Bezug auf Werbung eher untypische Medikamentenbranche ist.

3.3 Auch polylexikale Ökonyme tragen Züge natürlicher und künstlicher Benennungen. Das gilt für solche, die zum Teil aus wortfähigen, bedeutungstragenden Segmenten, zum Teil aus Ziffern, Kunst- oder Kurzwörtern bestehen. Solche Bildungen könnten ihrer Struktur nach als rechtsköpfige Komposita (Compu-Net) oder rechts- bzw. linksköpfige Syntagmen (Teledat 150 $P C I$ ) oder als Koppelung beider Strukturtypen (ISDN-Telefon T-Easy P520, Modem komplett Paket, T-ISDN mobil Tarif, ballOON Telecom Manager Plus, ein Modem) bestimmt werden. ${ }^{7}$ Ihre Glieder gehen jedoch meist kei-

${ }^{6}$ Vom Kurzwort unterscheidet sich das Kunstwort dadurch, dass es ein neues Wort darstellt und nicht die kürzere Variante einer Vollform (Kobler-Trill 1994, S. 129), auch wenn es wie das Kurzwort mitunter durch Kürzungsverfahren entsteht (Haribo, adidas, Eduscho).

7 In manchen Fällen sind zudem sowohl rechts- als auch linksköpfige Interpretationen der ganzen Fügung möglich: der/das Golf Cabriolet. 
ne eindeutige morphosyntaktische und/oder semantische Beziehung zueinander ein. Indiz dafür ist nicht zuletzt der mitunter völlig willkürliche Umgang der Namengeber mit der Schreibung. Zwischenräume signalisieren nicht in jedem Fall Wortgrenzen, sondern werden umfunktioniert. Spatium und Binnenmajuskel gelten heute ausdrücklich als Mittel zur „Onymisierung" (Stein 1999, S. 274) ${ }^{8}$ Während die Binnenmajuskel bei nicht regelgerechter Zusammenschreibung eine syntaktische Struktur andeutet (SkodaFabia, SkodaOktavia), ${ }^{9}$ verunklart umgekehrt das Spatium in komplexen Namen den Wortcharakter (AktivPlus Tarif). Diese Namen sind weder eindeutig monolexikal noch eindeutig polylexikal.

Zwischen natürlicher und künstlicher Benennungsbildung bewegen sich schließlich auch komplexe Namen, bei denen in der Regel eine sachlich bestimmte feste Reihenfolge ihrer Segmente in appositionsähnlicher Struktur üblich ist wie bei Fahrzeugbenennungen: Renault Twingo Enjoy ${ }^{10}$. Anders als bei Phraseologismen, auch bei den sog. teilbaren, ist jedes Glied separat namenwertig und referenzfähig: der Renault, der Twingo, der Enjoy.

\section{Wortbildung und Bedeutungsbildung}

Wortbildung und Bedeutungsbildung interferieren systematisch auf mindestens zweifache Weise, und zwar indem sie simultan an der Bildung komplexer Wörter beteiligt sind (Salamitaktik) oder indem sich aus einem komplexen Wort eine Konstituente mit der Bedeutung des komplexen Wortes verselbständigt (Gipfelkonferenz $>$ Gipfel). Im ersten Fall handelt es sich um konstitutive, im zweiten Fall um elliptische Bedeutungsbildung.

4.1 Unter konstitutiver Bedeutungsbildung wird die Bindung eines metaphorischen oder metonymischen Wortgebrauchs an einen Kompositionskontext verstanden", ich konzentriere mich auf den metaphorischen Gebrauch. Das jeweils figurativ verwendete Wort kann Erstglied (Spagettiträger, Etuikleid) oder auch Zweitglied des Kompositums sein (Datenautobahn, Blutbank). Für den Ausbau des Wortschatzes scheint die konstitutive Bedeutungsbildung außerordentlich wichtig zu sein. In einer vergleichenden

${ }^{8}$ Die Firma Volkswagen teilt auf die Frage nach den Gründen für die abweichende Schreibung von Angeboten wie Service Telefon, Unfall Schnelldienst, Fahrzeug Check, die man in ihren Fahrzeugbegleitprospekten findet, mit: „Bei den von Ihnen angesprochenen Beispielen handelt es sich um Eigennamen. Es werden hier Einrichtungen definiert, die im Einzelfall für unsere Kunden von großer Bedeutung sein können. Durch eine entsprechende Schreibweise soll eine höhere Aufmerksamkeit erreicht werden. Die besondere Schreibweise bestimmter Begriffe ist also Bestandteil der Werbung und somit Teil unserer Marketingstrategie." (Schreiben vom 25.01.1999).

${ }^{9}$ Eine ausführliche Funktionsbeschreibung der Binnenmajuskel liefert Stein 1999.

10 Von Munske als Phraseologismus eingestuft (1993, S. 494).

$"$ Terminus nach Fleischer 1996, S. 153, seine Beispiele sind Ölpest, grüne Lunge; zur metaphorischen Benennungsbildung Blank 1987, S. 159; auch Käge 1980. 
deutsch-schwedischen Untersuchung neuer Benennungen in Zeitungstexten entdeckt Inghult (1993, S. 159), dass innovative Metaphorik häufiger an Komposita gebunden vorkommt als an einen freien syntaktischen Kontext.

Der empirische Nachweis der Bindung der Bedeutungsbildung an den Kompositionskontext ist durch solche Korpusanalysen nur dann relativ zuverlässig zu erbringen, wenn ein Abgleich mit aktuellen Bedeutungswörterbüchern vorgenommen wird, denn die subjektive Wahrnehmung führt hierbei leicht in die Irre. Mit dem ersten Auftreten einer übertragenen Bedeutung im Kompositum ist offenbar sogleich auch die Potenz des Gebrauchs in der syntaktischen Fügung gegeben, nur werden beide Möglichkeiten nicht gleichermaßen genutzt. So fiel mir bei der Vorbereitung dieses Beitrages das anschauliche Kompositum Patchworkfamilie auf (,Familie, in der außer dem gemeinsamen Kind, den gemeinsamen Kindern, auch Kinder aus früheren Beziehungen der Mutter bzw. des Vaters oder beider Eltern leben', Duden 1999, S. 2870). Das schien ein treffendes Beispiel für konstitutive Bedeutungsbildung zu sein, zumal der Duden bei Patchwork kein metaphorisches Semem verzeichnet. Patchwork trägt als selbstständiges Lemma nur die Bedeutungen ,Handarbeitstechnik' und ,Produkt dieser Technik'. Von den Belegen aus 11 Millionen Sätzen im Leipziger Korpus „Deutscher Wortschatz“ musste ich mich allerdings eines Besseren belehren lassen: Sowohl okkasionelle Kompositionsmetaphern als auch metaphorischer Wortgebrauch in Syntagmen sind dort zahlreich gebucht; eine Auswahl: PatchworkBaukonzept, -Biographien, -Deutschland, -Ich, -Lebensläufe, -Wissen, -getue, -programme; Lebens-, Vokalpatchwork; Patchwork von Dienstleistungen, Patchwork der Stile, ein Patchwork zahlreicher Volksgruppen, Patchwork aus E- und U-Kunst, Patchwork an Beobachtungen und historischen Abrissen, Patchwork der Kinogeschichte, Patchwork der Nettigkeiten, akustisches, erzählerisches Patchwork. Sogar als Name einer Musikgruppe ist das Wort belegt. Patchworkfamilie ist demnach kein geeigneter Beleg für die konstitutive Bedeutungsbildung.

Nach der Wortbildungsaktivität der übertragenen Konstituente und deren Disponibilität im Syntagma ergeben sich drei Typen der interferierenden Benennungsbildung, die allerdings für das einzelne Semem keine zwingende chronologische Abfolge darstellen. Jedes figurative Semem kann auf jeder Stufe verharren und in dieser Verwendung usuell sein.

\section{Typ Salamitaktik}

Dieser Typ ist durch den singulären metaphorischen Gebrauch des Erst- bzw. Zweitgliedes gekennzeichnet. Meist ist nur ein Kompositum mit dieser Konstituente belegt; nicht dagegen der freie Gebrauch des fraglichen Wortes in der metaphorischen Bedeutung. Hierher gehören Buschzulage, Etuikleid, Reformstau, Reißverschlussverfahren, Spagettiträger. Singuläre Kompositionsmetaphern können sowohl lexikalisierte als auch okkasionelle Bildungen sein. Lexikalisierte Kompositionsmetaphern wie etwa Sackgasse, 
Sackbahnhof, die einen Gebrauch ihrer metaphorischen Konstituente in Syntagmen heute wohl nicht mehr zulassen, können in früheren Sprachstadien durchaus Ausdrucksalternativen zu Syntagmen gewesen sein. Das Grimmsche Wörterbuch verzeichnet noch bis Goethe Belege für den Gebrauch von Sack in der Bedeutung ,Straße, die nur einen Ausgang hat'. Das Lemma Sackgasse ist dort umschrieben mit ,eine strasze, die in einem sack endigt" (Grimm 1991, Spalte 1623).

\section{Typ Datenautobahn}

Die figurativ gebrauchte Konstituente dieses Typs ist stark wortbildungsaktiv, sie kommt in mehreren Komposita vor, ohne dass sie sich in ähnlicher Verbreitung als freies Lexem nachweisen ließe. Einzelvorkommen in syntaktischer Fügung sind allerdings nicht ausgeschlossen. Das Leipziger Korpus verzeichnet am 20.1. $2000 \mathrm{zu}$-autobahn, elektronische Verbindung': Breitbanddatenautobahn, BRD-Datenautobahn, Bundesdatenautobahn, US-Datenautobahn, Datensuperautobahn, Superdatenautobahn, Datenstadtautobahn, Devisenautobahn, Druckerautobahn, Erfahrungsautobahn, Informationsautobahn, Infoautobahn, Info-Autobahn, Informationssuperautobahn, Super-Infoautobahn, Investitionsautobahn, Kabelautobahn, Programmautobahn, Warenautobahn. Für den freien Gebrauch von Autobahn in dieser Bedeutung liegen mir keine Belege vor.

$\mathrm{Zu}$ diesem Typ gehören auch: -bank, -hai (Börsen-, Immobilien-, Geld-, Kredit, Miethai), -silo (Wohn-, Beamten-, Bücher-, Raketensilo), -telefon (Abfall-Telefon, Aids-Beratungstelefon, Anti-Gewalt-Telefon, BahncardServicetelefon, Baumtelefon, Beschwerde-Telefon, Bürgertelefon, Gesundheitstelefon, Reise-, Sprachtelefon), -wut (Arbeits-, Lese-, Putz-, Reform-, Tanz-, Zerstörungswut).

In diesen Fällen trägt das jeweils lautgleiche freie Wort (Bank, Hai usw.) nach den Angaben des Duden (1999) noch nicht die metaphorische Bedeutung, zumindest nicht usuell. Immerhin findet sich bei -wut doch ein Belegsatz mit Wut in der metaphorischen Verwendung: „Mit Wut (,großem Eifer, Arbeitswut") machten sie sich ans Werk", Duden 1999, 4572). Schu (1997, S. 69) zeigt am Beispiel -telefon, dass die Verbreitung des neuen Semems in selbstständiger Verwendung interessanterweise durch die Bindestrichschreibung wie in Grammatik-Telefon befördert werden kann.

\section{Typ Akademikerschwemme}

Die Bedeutungsbildung ist nicht an den Kompositionskontext gebunden. Reihenbildung bei den Komposita und Gebrauch der metaphorischen Bedeutung im Syntagma lassen sich gleichermaßen belegen. Das trifft z. B. zu auf-killer, -landschaft, -lawine, -mafia, -meer, -papst, -park, -sturm, -szene, -welle.

Einen Sonderfall stellen parallele Metaphorisierungen desselben Wortes dar, die sich in den verschiedenen Umgebungen semantisch unterscheiden: 
Schlüssel zum Erfolg ,Mittel für etwas', aber Schlüsselfrage, wichtige, zentrale Frage'.

4.2 Auch bei der zweiten Art der an Wortbildung gebundenen Bedeutungsbildung, der elliptischen oder syntagmatischen, entsteht Polysemie, nur setzt der Prozess strukturell-morphologisch als Reduktion ein. Der Sprecher reduziert ein komplexes Wort um eine Konstituente und gebraucht die verbleibende Konstituente in der Bedeutung des komplexen Ausgangswortes.

Dieses Verfahren wird sowohl bei komplexen Wörtern als auch bei Syntagmen (dann Reduktion um Wörter) zum „Auslöser“ von Bedeutungsbildung (Blank 1997, S. 285). Es führt entweder zu neuen Phraseologismen, wie Munske (1993, S. 503) beschreibt, oder zu meist polysemen Wörtern, wenn Komposita verkürzt werden. Nur Letzteres wird hier berücksichtigt. Bedingung für die Ellipse einer unmittelbaren Konstituente ist die morphosemantische Motiviertheit der Komposita. Sowohl Determinans (Schallplatte $>$ Platte) als auch Determinatum (Kornbranntwein $>$ Korn, Plattenbau > Platte) können entfallen.

An differenzierten empirischen lexikologischen Untersuchungen elliptischer Bedeutungsbildung auf Wortebene mangelt es noch. Wir wissen momentan kaum, wie intensiv das Verfahren okkasionell genutzt wird, in welchen Texten, in welcher Frequenz, und ob es bevorzugt mündlich oder schriftlich angewendet wird. Über die Lexikalisierungsaffinität der „Restwörter“" ist ebenfalls kaum etwas bekannt. Auch die Wortbildungslehre hat sich mit der elliptischen Bedeutungsbildung so gut wie nicht beschäftigt. Sie interessiert sich wohl für die formale Seite dieser Reduktion; die Semantik der Kürzungsprodukte dagegen fasst sie nicht als ihren Gegenstand auf. Henzen verweist das Phänomen ausdrücklich in die Zuständigkeit der Bedeutungslehre (Henzen 1965 , S. 264). Vermutlich wegen dieser unklaren Disziplinbindung wissen wir über die Wirksamkeit dieses Verfahrens im gegenwärtigen Deutsch so wenig.

Nach Wellanders historisch angelegter Untersuchung elliptischer Bedeutungsbildung liegt erst mit Blank (1997) eine ausführliche Arbeit dazu vor. Blank beschreibt am Beispiel romanischer Sprachen die Folgen elliptischer Bedeutungsbildung für das Lexikon, die auch auf das Deutsche übertragen werden können, wobei natürlich nicht jede referenzidentische Verwendung von Langform und Restglied in Texten zu elliptischer Bedeutungsbildung auf Dauer, d.h. zur Lexikalisierung eines neuen Semems führt. Eine grundlegende Bedingung für die Lexikalisierung ist wohl eine hohe Frequenz des komplexen Wortes (Blank 1997, S. 391).

Von den Blankschen Beobachtungen seien die beiden folgenden ausgewählt und an deutschsprachigen Beispielen illustriert.

(1) Es ist keineswegs so, dass das Kompositum nach der Usualisierung der elliptischen Form zwangsläufig ungebräuchlich wird, sondern Langform und Restwort bleiben meist nebeneinander in Gebrauch; es ist von Synonymie 
zwischen beiden Formen auszugehen. Diese Auffassung vertritt auch Kempcke, indem er die entsprechenden Wortpaare in seinem Wörterbuch als Synonyme ausweist, z. B. Bahn und Eisenbahn, Straßenbahn (Kempcke 2000, S. 98).

(2) Das zur Bedeutung des Restwortes hinzukommende Semem kann zu den bereits üblichen in unterschiedliche Beziehungen treten.

$\mathrm{Zu}$ (1): Die Durchsicht synchroner Wörterbücher erbringt für das Gegenwartsdeutsch ein stattliches Inventar solcher lexikalisierter Wortpaare, dazu ausgewählte Beispiele (nach LWB 1998):

Ellipse des Determinans:

(Nachrichten)Agentur, (Heirats)Antrag, (Rechts)Anwalt, (Haus/Schul)Aufgabe, (Eisen/Straßen)Bahn, (Luft)Ballon, (Farb/Fließ)Band, (Back/ Kuchen)Blech, (Kleider)Bügel, (Brillen/Fern/Opern)Glas, (Kühler)Grill, (Sicherheits)Gurt, (Augen)Höhle, (Herz)Infarkt, (Mannschafts)Kapitän, (Spiel)Kasino, (Kranken/Spar)Kasse, (Brief/Schau/Schub)Kasten, (Zünd)Kerze, (Müll)Kippe, (Rasier)Klinge, (Tür)Klinke; (Maschinen/Wagen/Fuhr)Park, (Blitz/Menschen/Schicksals/Strom)Schlag.

Ellipse des Determinatums:

Bass(geige/gitarre), Blitz(licht/lichtgerät), Bock(bier), Bund(eswehr), Gips(verband), Grund(besitz), Jumbo(-Jet), Kabel(fernsehen), Kali(salz), Korn(branntwein/schnaps), Schlag(anfall/baum), Weizen(bier).

Je nach ideolektaler und soziolektaler Situation der Sprecher kann es Verwendungsunterschiede geben. Vertrautheit mit den entsprechenden Denotaten oder referenzsichernde Kontext- oder Situationsbindung führen leicht zum Gebrauch der reduzierten Form. So mag Grill in der Bedeutung ,Kühlergrill' dem Nichtautofahrer eher ungeläufig sein, wie auch Park als ,Fuhrpark' deutlich soziolektal markiert ist. Platte als ,Plattenbau' ist besonders in jüngster Zeit im Zusammenhang mit dem Wohnungsleerstand als saloppe Insider-Benennung üblich geworden. Der Duden (1999) markiert auch Tochter ,Tochterfirma' als ,Jargon'.

$\mathrm{Zu} \mathrm{(2):} \mathrm{Auffällig} \mathrm{ist} \mathrm{eine} \mathrm{außerordentliche} \mathrm{Heterogenität} \mathrm{der} \mathrm{semantischen}$ Struktur des Restwortes. Die Beziehungen zwischen den Sememen beschreibt Blank (1997, S. 286 ff.) als hyperonymisch-hyponymisch oder metonymisch. Beispiele hierfür sind Gurt (1), Band zum Halten, Tragen', (2) , Gurt im Auto, Flugzeug', Blech (1) ,Material', (2) ,Gegenstand aus Blech'. Das scheinen jedoch keineswegs alle Möglichkeiten des Bedeutungsverhältnisses zu sein, vgl. der (Swimming)Pool, das Pool(billard), der/das Korn als Homonyme (wenn man Genusdifferenz als Merkmal für Homonymie akzeptiert), Tochter ,Kind ' und ,Teil eines Unternehmens' in metaphorischer Beziehung.

Im Deutschen entsteht auch durchaus nicht immer ein polysemes Wort als Folge der Ellipse einer Konstituente, z. B. dann nicht, wenn das Zweitglied des Kompositums ein verdeutlichender Oberbegriff ist wie bei Kali(salz). 
Fast in allen Fällen sind die Restwörter Konkreta, was die Frage nach generellen Präferenzen elliptischen Wortgebrauchs aufwirft. Komposita in fixierten Kollokationen wie Schweigepflicht in ärztliche Schweigepflicht können z.B. nicht ohne weiteres elliptisch verkürzt werden (Wellmann 1993, S. $161 \mathrm{ff}$.). Weniger signifikant ist möglicherweise der Zusammenhang zwischen dem Grad der Polysemie des verbleibenden Wortes und der Neigung zu elliptischer Verkürzung. Stark ausgeprägte Polysemie eines Gliedes scheint die Neigung zur Ellipse nicht zu stören, wovon beispielweise die stark polysemen Restglieder Bahn, Schlag zeugen. Benennungskonkurrenz verhindert Kürzung ebenfalls nicht, vgl. das neuere Kabel (für ,Fernsehanschluss durch Verkabelung') neben Kabelfernsehnetz, Kabelnetz, Kabelfernsehen, Kabelanschluss, Kabel-TV in der Verwendung ,Habt ihr zu Hause Kabel? ' (Duden $(1999,2022)$ oder Tochter(betrieb/firma/gesellschaft/unternehmen, aber nichtelliptisch Tochtergeschwulst, Tochtergeneration, Tochterzelle u. A.).

Weitere Untersuchungen sind ebenso erforderlich zu den Lexikalisierungsbedingungen und zur Entwicklung der Synonymie auf längere Sicht. Bei Henzen (1965, S. 264) findet man Beispiele, deren Langform heute entweder ungebräuchlich geworden ist (Kauf/Krämer)Laden oder deren Restglieder nicht (mehr) die Bedeutung der ganzen Form (Gieß/Kaffee)Kanne haben, sondern nur mehr hyperonymisch zum Kompositum zu interpretieren sind. Der Rückgang des Gebrauchs von Ampel für Ampelkoalition schon kurz nach Aufkommen der kürzeren Form infolge der politischenVeränderungen oder auch von Eisen ,Bügeleisen', vermutlich ebenfalls wegen Sachwandels, das der Duden (1999) nicht mehr mit dem Semem ,Bügeleisen“ verzeichnet, verweisen auf die pragmatische Bedingtheit gerade dieser Synonymierelation.

\section{Ausblick}

Abschließend sei am Beispiel von Wortbildung und Phraseologisierung auf eine weitere, ganz andere Art des Zusammenwirkens zweier Nominationsverfahren verwiesen. Das Verhältnis zwischen Wortbildung und Phraseologisierung wird bislang vor allem als ,Arbeitsteilung“ beschrieben (Fleischer 1992; 1997), indem jedem Nominationsverfahren eine spezifische morphosyntaktische und funktionale Domäne beim Wortschatzausbau und im Sprachgebrauch zugewiesen wird. Wählt man nun die figurative Bedeutungsbildung als Ansatzpunkt und vergleicht die entsprechenden Möglichkeiten in Wortbildung und Phraseologie, zeigen sich deutlich Gemeinsamkeiten. Burger (1998, S. $81 \mathrm{ff}$.) schlägt in Anlehnung an die kognitive Metaphernforschung vor, bei der Beschreibung dieser Gemeinsamkeiten mit dem Begriff des metaphorischen Modells zu arbeiten. Ein metaphorisches Modell kann danach sowohl als Wort-Metapher als auch als metaphorischer Phraseologismus realisiert sein, wie etwa in Radarfalle - jmdm. eine Falle 
stellen, in eine Falle locken, in eine Falle geraten; Bilderbuchehe, -ehemann, -karriere, -landung, -tor, -sommer, -wetter - ein X wie im/aus dem Bilderbuch, Ellenbogenfreiheit, -gesellschaft, -mensch, -taktik - seine Ellenbogen gebrauchen, keine Ellenbogen haben. Diese Beziehungen deuten auf ein neues, noch weitgehend unbearbeitetes Feld künftiger Nominationsforschung.

\section{Literatur}

\section{Wörterbücher und Quellen}

Deutscher Wortschatz. Korpus des Instituts für Informatik an der Universität Leipzig.

Duden (1999): = Duden. Das große Wörterbuch der deutschen Sprache in 10 Bänden. 3. völlig neu bearb. und erw. Aufl. Hg. v. Wissensch. Rat der Dudenredaktion. Mannheim u. a.

Grimm 1991 = Deutsches Wörterbuch von Jakob und Wilhelm Grimm. 33 Bände. Leipzig 1854-1961. Nachdruck als Taschenbuchausgabe Bd. 14. dtv München.

LWB (1998): = Götz, Dieter/Haensch, Günther/Wellmann, Hans (Hg.): Langenscheidts Großwörterbuch Deutsch als Fremdsprache. München.

Kempcke, Günter u.a. (2000): Wörterbuch Deutsch als Femdsprache. Berlin/New York.

\section{Sonstige Literatur}

Blank, Andreas (1997): Prinzipien des lexikalischen Bedeutungswandels am Beispiel der romanischen Sprachen. Beihefte zur Zeitschrift für Romanische Philologie. Bd. 285. Tübingen.

Burger, Harald (1998): Phraseologie. Eine Einführung am Beispiel des Deutschen. Berlin.

Eichinger, Ludwig M. (1997): Weltansicht in Wörtern. Vom Zweck und Nutzen verbaler Wortbildung. In: Simečková, A./Vachková, M. (Hg.): Wortbildung. Theorie und Anwendung. Praha. S. 2441.

Eisenberg, Peter (1998): Grundriß der deutschen Grammatik. Bd. 1. Das Wort. Stuttgart/ Weimar.

Erben, Johannes (1993): Einführung in die deutsche Wortbildungslehre. Berlin.

Fleischer, Wolfgang (1989): Nomination und unfeste nominative Ketten. In: Beiträge zur Erforschung der deutschen Sprache 9, S. 13-27.

Fleischer, Wolfgang (1992): Konvergenz und Divergenz von Wortbildung und Phraseologisierung. In: Korhonen, Jarmo (Hg.): Phraseologie und Wortbildung - Aspekte der Lexikonerweiterung. Tübingen. S. 53-65.

Fleischer, Wolfgang (1996): Phraseologische, terminologische und onymische Wortgruppen als Nominationseinheiten. In: Knobloch, Clemens/Schaeder, Burkhard ( $\mathrm{Hg}$.): Nomination - fachsprachlich und gemeinsprachlich. Opladen. S. 147-170.

Fleischer, Wolfgang (1997): Das Zusammenwirken von Wortbildung und Phraseologisierung in der Entwicklung des Wortschatzes. In: Wimmer, Rainer/Berens, Franz Josef (Hg.): Wortbildung und Phraseologie. Tübingen. S. 9-24.

Fleischer, Wolfgang/Barz, Irmhild (1995): Wortbildung der deutschen Gegenwartssprache. Tübingen.

Gréciano, Gertrud (1997): Zur Festigung von Phraseologie. Eine Merkmalanalyse. In: Barz, Irmhild/Schröder, Marianne (Hg.): Nominationsforschung im Deutschen. Festschrift für Wolfgang Fleischer zum 75. Geburtstag. Frankfurt/M.S. 167-175. 
Große, Rudolf (1997): Nomination im Althochdeutschen. In: Barz, Irmhild/Schröder, Marianne (Hg.): Nominationsforschung im Deutschen. Festschrift für Wolfgang Fleischer zum 75. Geburtstag. Frankfurt/M. S. 15-21.

Henzen, Walter (1965): Deutsche Wortbildung. Tübingen.

Inghult, Göran (1993): Über die Möglichkeiten der Erweiterung des Lexembestandes im Deutschen und Schwedischen. In: Göteborger Germanistische Forschungen 35, S. 155-168.

Janich, Nina (1999): Werbesprache. Ein Arbeitsbuch. Tübingen.

Käge, Otmar (1980): Motivation: Probleme des persuasiven Sprachgebrauchs, der Metapher und des Wortspiels. Göppingen.

Kalverkämper, Hartwig (1978): Textlinguistik der Eigennamen. Stuttgart.

Knobloch, Clemens/Schaeder, Burkhard (1996): Nomination - fachsprachlich und gemeinsprachlich. Ein Vorwort. In: Knobloch, Clemens/Schaeder, Burkhard (Hg.): Nomination - fachsprachlich und gemeinsprachlich. Opladen. S. 7-19.

Kobler-Trill, Dorothea (1994): Das Kurzwort im Deutschen. Eine Untersuchung zu Definition, Typologie und Entwicklung. Tübingen.

Koß, Gerhard (1990): Namenforschung. Eine Einführung in die Onomastik. Tübingen.

Koß, Gerhard (1992): Tabs und Tablinen. Zur Wortbildung bei Medikamentennamen. In: Große, Rudolf/Lerchner, Gotthard/Schröder, Marianne (Hg.): Beiträge zur Phraseologie, Wortbildung, Lexikologie. Frankfurt/M. S. 145-166.

Lerchner, Gotthard (1990): Mustermischung und Sprachausgleich im trivialliterarischen Diskurs des 18. Jahrhunderts. In: ZGL 18.3, S. 261-272.

Lerchner, Gotthard (1997): Nomination und Semiose. Zur Explikation ihrer kulturellkommunikativen Geprägtheit. In: Barz, Irmhild/Schröder, Marianne (Hg.): Nominationsforschung im Deutschen. Festschrift für Wolfgang Fleischer zum 75. Geburtstag. Frankfurt/M.S. 147-155.

Lipka, Leonhard (1994): Wortbildung, Metapher und Metonymie - Prozesse, Resultate und ihre Beschreibung. In: Staib, Bruno (Hg.): Münstersches Logbuch zur Linguistik 5. Münster. S. 1-15.

Matussek, Magdalena (1994): Wortneubildung im Text. Hamburg.

Motsch, Wolfgang (1999): Deutsche Wortbildung in Grundzügen. Berlin/New York.

Munske, Horst Haider (1990): Über den Wandel des deutschen Wortschatzes. In: Besch, Werner (Hg.): Deutsche Sprachgeschichte. Grundlagen, Methoden, Perspektiven. Festschrift für Johannes Erben zum 65. Geburtstag. Frankfurt/M.S. 387-401.

Munske, Horst Haider (1993): Wie entstehen Phraseologismen? In: Mattheier, Klaus J. u. a. (Hg.): Vielfalt des Deutschen. Festschrift für Werner Besch. Frankfurt/M. S. 481-516.

Naumann, Bernd (1986): Einführung in die Wortbildungslehre des Deutschen. Tübingen.

Platen, Christoph (1997): „Ökonymie“. Zur Produktnamen-Linguistik im Europäischen Binnenmarkt. Tübingen.

Platen, Christoph (2000): Köpi, Kelts und Knusperone. Morpheme in den Zeiten der Marktwirtschaft. In: Barz, Irmhild/Fix, Ulla/Schröder, Marianne (Hg.): Praxis- und Integrationsfelder der Wortbildungsforschung. Heidelberg. S. 239-251.

Schu, Josef (1997): -telefon. Lexikalischer Wandel durch Wortbildung. In: Deutsche Sprache 25, S. 54-82.

Seebold, Elmar (1981): Etymologie. Eine Einführung am Beispiel der deutschen Sprache. München.

Stein, Stephan (1999): Majuskeln im WortInnern. Ein neuer graphostilistischer Trend für die Schreibung von Komposita in der Werbesprache. In: Muttersprache 109, S. 261-278.

Stoll, Kai-Uwe (1999): Markennamen. Sprachliche Strukturen, Ähnlichkeit und Verwechselbarkeit. Frankfurt/M. 
Voiggt, Gerhard (1984): Markennamen. Die fremden Alltagswörter. In: Praxis Deutsch 67, S. 63-70.

Welllander, Erik (1917; 1923; 1928): Studien zum Bedeutungswandel im Deutschen. In: Uppsala Universitets Årsskrift.

Welllmann, Hans (1993): Die Bedeutungen der Nominalkomposita. In: Wellmann, Hans (Hg.): Synchrone und diachrone Aspekte der Wortbildung im Deutschen. Heidelberg. S. $147-168$.

Welllmann, Hans (1998): Die Wortbildung. In: Duden Band 4. Grammatik der deutschen Gegenwartssprache. 6., neu bearbeitete Auflage. Mannheim u. a. S. 408- 557. 